\title{
Risk of obstetric anal sphincter injury among women who birth vaginally after a prior caesarean section: A state-wide cohort study
}

\author{
Jessica Uebergang ${ }^{1}$, Richard Hiscock ${ }^{2}$, Roxanne Hastie ${ }^{2}$, Anna Middleton ${ }^{2}$, Natasha \\ Pritchard $^{1}$, Susan Walker ${ }^{3}$, Stephen Tong ${ }^{3}$, and Anthea Lindquist ${ }^{2}$ \\ ${ }^{1}$ Mercy Hospital for Women \\ ${ }^{2}$ University of Melbourne, Mercy Hospital for Women \\ ${ }^{3}$ University of Melbourne
}

September 25, 2021

\begin{abstract}
Objective: Vaginal birth after caesarean (VBAC) has been suggested to be associated with an increased risk of obstetric anal sphincter injury (compared with primiparous women who birth vaginally). However, prior studies have been small, or used outdated methodology. We set out to validate whether the risk of obstetric anal sphincter injury among women having their first VBAC is greater than that among primiparous women having a vaginal birth. Design: State-wide retrospective cohort study. Setting: Victoria, Australia. Population: All births $(455,000)$ between 2009-2014. Methods: The risk of severe perineal injury between first vaginal birth and first vaginal birth after previous caesarean section was compared, after adjustment for potential confounding variables. Covariates were examined using logistic regression for categorical data and Wilcoxon rank-sum test for continuous data. Missing data were handled using multiple imputation; the analysis was performed using regression adjustment and Stata v16 multiple imputation and teffects suites. Results: Women having a VBAC $(\mathrm{n}=5,429)$ were significantly more likely than primiparous women $(\mathrm{n}=123,353)$ to sustain a $3 \mathrm{rd}$ or 4 th degree tear during vaginal birth $(7.1 \mathrm{vs} 5.7 \%$, $\mathrm{p}<0.001)$. After adjustment for mode of birth, body mass index, maternal age, infant birthweight, episiotomy and epidural, there was a $21 \%$ increased risk of severe perineal injury (relative risk $1.21(95 \%$ CI $1.07-1.38)$ ). Conclusions: Women having their first vaginal birth after caesarean section have a significant increased risk of sustaining a 3rd or 4th degree tear, compared with primiparous women having a vaginal birth. Patient counselling and professional guidelines should reflect this increased risk.
\end{abstract}

\section{INTRODUCTION}

Obstetric anal sphincter injury reflects significant perineal trauma during childbirth and involves injury of the anal sphincter complex (1). While many heal well after primary repair, the impact of this injury for some can be lifelong with long-term symptoms persisting well beyond the birth (2-4). These symptoms include perineal pain, dyspareunia, defecatory dysfunction and faecal incontinence and they can significantly impact a woman's quality of life and emotional well-being $(3,5)$.

The incidence of obstetric anal sphincter injury amongst primiparous women varies but is reported to be $5.3 \%$ in Australia, $6.1 \%$ in the UK and up to $16 \%$ in the US $(1,6,7)$. Risk factors include nulliparity, advanced maternal age, and intrapartum factors that might plausibly be associated with pelvic trauma such as infant birthweight $>4000 \mathrm{~g}$ and instrumental vaginal birth (8-11).

In most Western jurisdictions, up to $30 \%$ of primiparous women give birth via caesarean section $(6,12)$. For women in their second pregnancy, VBAC is an increasingly desired mode of birth, with successful VBAC associated with a quicker post-partum recovery $(13,14)$.

However, compared with vaginal birth in primiparous women, there is emerging evidence suggesting successful 
VBAC is also associated with an increased risk of obstetric anal sphincter injury $(9,10,15)$. Past studies in this field have been limited by small cohort sizes, lack of broader generalisability (limited to a single hospital cohort) or outdated methodology (such as a reliance on complete case records only, which may lead to ascertainment bias) (16).

Prior studies have consistently suggested that women having a VBAC are up to 1.4-fold more likely to sustain significant perineal injury compared with their primiparous counterparts $(8,9,15)$. And yet, the risk of perineal injury is still only peripherally acknowledged in many professional guidelines $(17,18)$ and is not routinely included in antenatal counselling of women considering a VBAC. Using a large cohort of women who achieved a VBAC for their second birth and employing advanced statistical methodology, our study aimed to rigorously validate a possible association between VBAC and significant perineal injury.

\section{METHODS}

\section{Study design}

We performed a retrospective cohort study including 116,047 women who gave birth in the Australian state of Victoria between 2009 and 2014. Validated data were obtained from the Consultative Council on Obstetric and Paediatric Mortality and Morbidity (CCOPMM) for all births in Victoria. CCOPMM is the central agency that collects and validates data on obstetric and perinatal outcomes within the state (19-21). These data are populated by state-wide hospital documentation of outcomes by midwives caring for the patients at the time of birth.

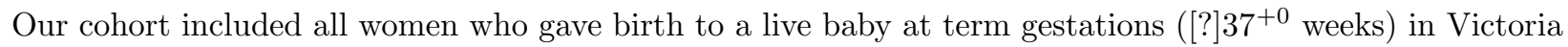
from $1^{\text {st }}$ Jan 2009 to $31^{\text {st }}$ Dec 2014 . Women were excluded if they had missing or incomplete gestational age data, missing or implausible birthweight $(<500 \mathrm{~g}$ or $>6500 \mathrm{~g})$, gave birth pre-term $(<37$ weeks) or had a stillborn baby (Figure 1). The primary exposure of interest was the first vaginal birth after one previous caesarean section. Women were therefore excluded if they did not fit the inclusion criteria for a) cases: multiparous women having had only one previous caesarean section followed by a vaginal birth, or b) controls: primiparous women having their first vaginal birth. The primary outcome of interest was documented obstetric anal sphincter injury ( $3^{\text {rd }}$ or $4^{\text {th }}$ degree tear).

\section{Statistical analysis}

We considered potential confounders between $3^{\text {rd }}$ and $4^{\text {th }}$ degree tear and VBAC status to be: maternal age (recorded to the nearest year at prenatal booking and centred at 30 years), mode of birth (as a three level nominal variable: normal vaginal birth, forceps vaginal birth and ventouse/vacuum vaginal birth), completed week of term gestational age (37-42 weeks), birthweight (continuous in grams), episiotomy (binary yes/no), BMI (continuous) and epidural analgesia (binary yes/no). Raw data for the outcome and covariates were presented as number (\%) or mean (SD) by exposure status. The proportion of missing data was documented for each covariate (Table 1).

The distribution of covariates between exposure groups was examined using univariable logistic regression for categorical data and Wilcoxon rank-sum test for continuous data, based upon non-missing data. The same tests were repeated as a sub-analysis, looking at the distribution of covariates between women who had a VBAC and had a $3^{\text {rd }}$ or $4^{\text {th }}$ degree tear, and women who had a VBAC but no significant perineal injury.

The adjusted analysis consisted of two parts: multiple imputation of missing covariate data, followed by regression adjustment analysis based upon potential outcome means (POM). We performed multiple imputation consisting of fully conditional specification (FCS) using a predictive mean model for continuous, logistic model for binary and multinominal model for unordered categorical covariates. The imputation model included outcome, exposure and all covariates used in the analysis model. There were no additional auxiliary variables available for inclusion. The number of imputed datasets (20) was set above the highest percentage of missingness and considered adequate if the Monte Carlo error was less than $10 \%$ of regression 
coefficient standard errors. Standard imputation diagnostics included: graphical assessment of convergence; comparison of distributional shape between both imputed (complete) and observed (non-imputed) datasets; and graphical and numerical imputed values and observed values.

The analysis was then performed on each of the 20 imputed datasets, using Regression Adjustment (RA) to derive a pooled estimate of potential outcome means (POM), Risk difference (RD) and Relative risk (RR) with associated $95 \%$ confidence limits adjusted for imputation using Rubin's rules. Regression adjustment estimates the POM separately for both exposure groups and these are then used to derive RD and RR estimates. The model will produce unbiased estimates if both covariate relationships are correctly specified, and no important confounders have been omitted.

Sensitivity analysis was performed using the same regression adjustment model for two complete case cohorts (including and excluding BMI and analgesia in the covariate list). Unadjusted RD and RR are also presented. Analysis was performed using Stata v16, including the multiple imputation and teffects suites. The significance level was two-sided and set at 0.05 .

\section{Ethics}

Ethical approval was obtained from the Mercy Hospital for Women Human Research Ethics Committee (\#2019-01). Because this was a retrospective study using archived de-identified data only, obtaining individual patient consent was not considered to be necessary by the ethical review board.

\section{RESULTS}

Our state-wide cohort included 455,455 births. After exclusions, there were 129,964 births for analysis: 123,535 primiparous women who birthed vaginally and 5,429 women who birthed vaginally for the first time after one previous caesarean section (Figure 1). Vaginal birth for both groups included those who birthed with the assistance of ventouse or forceps.

There were no missing data on VBAC status (exposure); $11.1 \%$ of the entire cohort had missing perineal tear data (outcome). There was no difference in distribution of missing outcome data ( $3^{\text {rd }}$ or $4^{\text {th }}$ degree tears) between exposure groups ( $10.1 \%$ in controls vs $9.3 \%$ in cases; $\mathrm{p}=0.09$ ). Imputation of missing outcome data was not performed (as no auxiliary variables) and so all cases with missing outcome data were excluded from final analysis. There were 116,047 births remaining for analysis. The distribution of covariates, including missingness, is presented in Table 1.

Compared with primiparous women having a vaginal birth, women having a VBAC were more likely to be older ( $>30$ years, $\mathrm{p}<0.001$ ), to have a slightly higher body mass index (mean 25.4 vs $24.7, \mathrm{p}<0.001$ ), to give birth between 38 and 40 completed weeks $(\mathrm{p}<0.001)$, to have a ventouse birth $(23.6$ vs $21.3 \%, \mathrm{p}<0.001)$ and for their baby to have a higher birthweight (mean birthweight 3452.7 vs $3377.3 \mathrm{~g}, \mathrm{p}<0.001$; incidence of birthweight [?]4000g 10.8 vs $8.2 \%, \mathrm{p}<0.001$ ) (Table 1). The VBAC group were less likely to have a spontaneous (unassisted) vaginal birth ( 56.7 vs $59.1 \%, \mathrm{p}<0.001)$ and less likely to have an intrapartum epidural ( 30.2 vs $36.0 \%, \mathrm{p}<0.001)$. There was no difference between the exposure groups in the likelihood of having an episiotomy ( 45.0 vs $46.2 \%, \mathrm{p}=0.18$ ).

Women having a VBAC were significantly more likely than primiparous women to sustain a $3^{\text {rd }}$ or $4^{\text {th }}$ degree tear during vaginal birth $(7.1$ vs $5.7 \%, \mathrm{p}<0.001)$. A sub-analysis examining only women who had a VBAC demonstrated that those who sustained a $3^{\text {rd }}$ or $4^{\text {th }}$ degree tear were more likely to have had a forceps birth (31.3 vs $18.8 \%, \mathrm{p}<0.001)$ and a baby with a birthweight [?]4000g (15.9 vs $10.4 \%, \mathrm{p}<0.001)$, and less likely to have had an episiotomy (33.6 vs $45.9 \%, \mathrm{p}=0.001$ ) (Table 2 ).

Unadjusted analysis produced a relative risk for women having a VBAC of $3^{\text {rd }}$ or $4^{\text {th }}$ degree tear of 1.24 (95\% CI 1.12 to 1.38 ) and a risk difference of $1.39 \%$ (95\% CI 0.66 to 2.12). The regression adjustment estimates were pooled over the 20 imputed datasets providing an adjusted relative risk amongst cases of 1.21 (95\% CI 1.07 to 1.38 ) and a risk difference of $1.22 \%$ (95\%CI 0.35 to 2.1). Sensitivity analysis was performed on complete case cohorts, which produced similar adjusted relative risks of 1.19 (95\% CI 1.03 to 1.38 ) when 
both BMI and analgesia were included, and 1.23 (95\%CI 1.09 to 1.39) when BMI and analgesia were both excluded (Table 3 ).

\section{DISCUSSION}

\section{Main findings}

Our study found that women having a vaginal birth after one previous caesarean section were $21 \%$ more likely than primiparous women having a vaginal birth to sustain a $3^{\text {rd }}$ or $4^{\text {th }}$ degree tear $(\mathrm{RR} 1.21,95 \% \mathrm{CI}$ 1.07-1.38). Given the baseline risk is not low (6.1\% in the UK for instance), this increase may be clinically significant. These findings indicate a level of risk that women should be made aware of during antenatal counselling if a VBAC is being considered. Across the different statistical models, the relative risk estimates were similar, all suggesting a significant increase in the incidence of $3^{\text {rd }}$ or $4^{\text {th }}$ degree tears associated with VBAC.

Our findings highlight the characteristics of women attempting a VBAC, and of those at increased risk of $3^{\text {rd }}$ and $4^{\text {th }}$ degree tears. These findings are in keeping with studies from comparable settings around the world. Women having a VBAC in our Victorian cohort were more likely to be older, have an instrumental birth and have a baby with higher birthweight. Those that sustained a $3^{\text {rd }}$ or $4^{\text {th }}$ degree tear during VBAC were more likely to have had a forceps birth, a baby of birthweight [?]4000g and less likely to have had an episiotomy. These factors are all known to increase the risk of $3^{\text {rd }}$ and $4^{\text {th }}$ degree tears $(9,22,23)$, with forceps considered a major risk factor for significant perineal injury (24-26).

In 2014, Hehir et al published results of a study from one large tertiary referral hospital in Dublin that concurred with our findings, that women having a VBAC were more likely to be older and have a baby with higher birthweight (consistent with being multiparous), and have an instrumental birth (10). Women who sustained a significant perineal injury during successful VBAC were also more likely to have had a forceps (27). A study conducted in Southampton, UK, between 2004-2014 looked at the maternal, intrapartum and neonatal factors associated with $3^{\text {rd }}$ or $4^{\text {th }}$ degree tear amongst 1,375 secundiparous women having a VBAC (8). They found that advanced maternal age, higher birthweight and an urgent category of first caesarean section were associated with an increased risk of $3^{\text {rd }}$ or $4^{\text {th }}$ degree tear (8). Episiotomy (right mediolateral) was found to be protective, consistent with the findings of ours and other studies (28-31).

Another UK-based study by Jardine et al examined a cohort of 9,993 secundiparous women having a VBAC and excluded any women with missing data (16). Whilst confirming the above findings, this study also suggested that women having a VBAC were more likely to experience a shoulder dystocia at vaginal birth, and that the increased risk of perineal injury following VBAC was restricted to women who had had an emergency primary caesarean section (16).

Other studies have hypothesised why women having a VBAC are at increased risk of $3^{\text {rd }}$ and $4^{\text {th }}$ degree perineal tears. Proposed mechanisms range from the mismatch between more propulsive uterine contractions in the multigravida VBAC cohort, coupled with a 'nulliparous perineum' $(32,33)$, through to relative cephalopelvic disproportion as the indication for first caesarean section affecting the passage of the fetus and leading to anal sphincter injury in subsequent births for these women $(9,11,32)$. Further, as demonstrated by ours and a number of studies, women attempting a VBAC are more likely to have an operative vaginal birth (10),

likely due to concern from the accoucheur about prolonged second stage and the risk of uterine rupture and fetal compromise $(8,34)$. This lower threshold for operative birth ultimately increases the risk of perineal injury. Irrespective of the mechanism, there is now convincing evidence that VBAC is associated with an increased risk of significant perineal injury, and education and prevention strategies should be directed toward this group.

\section{Study strengths and limitations}

Our study has several strengths. We used a large, state-wide cohort derived from 5 years of routinely collected 
data in Australia, to explore the association between VBAC and significant perineal injury. Whilst there is a growing body of published evidence recognising VBAC as a risk factor for $3^{\text {rd }}$ and $4^{\text {th }}$ degree tear, ours is one of the largest, most recently reported studies to explore this association and provides a more rigorous, updated approach to adjusted analyses than the existing published studies.

The main limitation of our study is the use of retrospective data, which can produce biased estimates of risk due to missing data and limited covariate detail. However, we thoroughly explored the pattern of missing data, specifically for body mass index and epidural for which $>10 \%$ of data were missing. It was important that data describing these two variables were appropriately accounted for, since high body mass index is thought to be protective against significant perineal injury $(35,36)$ and epidural increases the likelihood of instrumental birth which is a risk factor for $3^{\text {rd }}$ and $4^{\text {th }}$ degree tear $(10,37,38)$.

In contrast to other studies (16), our study accounted for missing data and our model was carefully planned and scrutinised. We attempted to overcome the limitations of routine data and produce the least biased results by imputing missing data, analysed for bias with appropriate diagnostic techniques, and performing sensitivity analyses with non-missing data. The main limitation for our multiple imputation was the absence of auxiliary variables. However, this is a recognised limitation of using routinely collected population data, and not a limitation of our analysis per se. All adjusted models, including complete case models, produced estimates within close proximity of each other, diminishing the likelihood that our final estimate of risk is misleading.

\section{Interpretation}

Our study has strong clinical relevance. We set out to validate the association between VBAC and significant perineal injury using a large cohort, and have demonstrated with updated methodology that women having a VBAC are $21 \%$ more likely than primiparous women to sustain a $3^{\text {rd }}$ or $4^{\text {th }}$ degree tear during vaginal birth. Current antenatal counselling and patient education for women attempting a VBAC typically focusses on the risk of uterine rupture and subsequent fetal complications $(17,18)$, often without any reference to the increased risk of obstetric anal sphincter injury. In light of our findings, we feel the evidence now convincingly demonstrates an increased risk of significant perineal injury associated with VBAC. We suggest that this risk should be included in the antenatal counselling of women considering a VBAC and be acknowledged in the guidelines of peak professional bodies such as the Royal Australian and New Zealand College of Obstetricians and Gynaecologists and the Royal College of Obstetricians and Gynaecologists.

Our study is well-timed with many Western jurisdictions introducing initiatives to prevent and reduce the occurrence of $3^{\text {rd }}$ and $4^{\text {th }}$ degree tears. Through measures such as timely mediolateral episiotomy and Manual Perineal Protection, the OASI Care Bundle, introduced throughout the UK in 2016-2017, was shown to significantly reduce the risk of $3^{\text {rd }}$ and $4^{\text {th }}$ degree tears (aOR 0.80; 95\%CI 0.65-0.98) in participating units (39). In Victoria, Australia, there is a current state-wide initiative to reduce the rate of $3^{\text {rd }}$ and $4^{\text {th }}$ degree tears amongst all women having a vaginal birth (the Better births for women collaborative), instigated in 2019 by the peak safety and quality institution, Safer Care Victoria (40). Neither the OASI Care Bundle in the UK or the Better Births initiative in Australia identified women having VBAC as a high-risk group, however our findings suggest that these women should be prioritised in such initiatives.

Our intention in pursuing this study was not to dissuade either patients or healthcare providers from supporting women attempting a vaginal birth after caesarean section, but rather to make antenatal counselling more comprehensive, birth management decisions better informed and to help optimise intrapartum care. There are myriad benefits associated with successful, uncomplicated VBAC, some of which include expedited post-birth recovery and improved breastfeeding initiation $(41,42)$. Vaginal birth after caesarean section also avoids the well-established risks related to repeat caesarean section delivery, such as significant bleeding, development of abdominal adhesions, increased future risk of placenta praevia, disorders of placental adherence and unnecessary iatrogenic preterm birth in the setting of threatened preterm labour $(43,44)$.

\section{Conclusion}


There are many social, economic and medical benefits of successful vaginal birth following caesarean section. Our study has confirmed that women considering a vaginal birth after caesarean section should be counselled about the increased risk of $3^{\text {rd }}$ and $4^{\text {th }}$ degree tears, and midwives and obstetricians working in the birthsuite setting should be appropriately educated about this increased risk.

\section{Acknowledgements:}

"We are grateful to CCOPMM for providing access to the data used for this project and for the assistance of the staff at Safer Care Victoria. The conclusions, findings, opinions and views or recommendations expressed in this paper are strictly those of the author(s). They do not necessarily reflect those of CCOPMM."

Disclosure of interests: None to declare.

Contribution to authorship: AL, JU and ST devised the study. JU, AL and NP obtained ethical approval and the data used for the study. AL and RJH devised the analysis plan, performed the analyses and wrote the Methods and Results sections of the manuscript. JU and AL wrote the manuscript. ST, RH and SW provided oversight and review of the analysis, results and manuscript on multiple occasions prior to submission for publication.

Details of ethical approval: Ethical approval was obtained from the Mercy Hospital for Women Human Research Ethics Committee (\#2019-01). Because this was a retrospective study using archived de-identified data only, obtaining individual patient consent was not considered to be necessary by the ethical review board.

Funding: Doctors Lindquist, Hastie, and Tong receive salary support from the National Health and Medical Research Council. Dr Prichard receives a scholarship from the National Health and Medical Research Council.

\section{Figures and Tables:}

Figure 1: Consort diagram of study population

Table 1. Participant characteristics by VBAC exposure

Table 2. Characteristics of women who had a VBAC, by perineal tear status

Table 3. Results of regression adjustment and sensitivity analyses

\section{References}

1. Royal College of Obstetricians and Gynaecologists. The Management of Third- and Fourth-Degree Perineal Tears. Green-top Guideline No29. 2015.

2. LaCross A, Groff M, Smaldone A. Obstetric anal sphincter injury and anal incontinence following vaginal birth: a systematic review and meta-analysis. J Midwifery Womens Health. 2015;60(1):37-47.

3. Leeman L, Rogers R, Borders N, Teaf D, Qualls C. The Effect of Perineal Lacerations on Pelvic Floor Function and Anatomy at 6 Months Postpartum in a Prospective Cohort of Nulliparous Women. Birth. 2016;43(4):293-302.

4. Fernando RJ, Sultan AH, Radley S, Jones PW, Johanson RB. Management of obstetric anal sphincter injury: a systematic review \& national practice survey. BMC Health Services Research. 2002;2(1):1-10.

5. Priddis H, Schmied V, Dahlen H. Women's experiences following severe perineal trauma: a qualitative study. BMC Womens Health. 2014;14(1):32.

6. Health AIo, Welfare. Australia's mothers and babies 2018 - in brief. Canberra: AIHW; 2020.

7. Lowder JL, Burrows LJ, Krohn MA, Weber AM. Risk factors for primary and subsequent anal sphincter lacerations: a comparison of cohorts by parity and prior mode of delivery. Am J Obstet Gynecol. 2007;196(4):344.e1-5. 
8. D'Souza JC, Monga A, Tincello DG. Risk factors for obstetric anal sphincter injuries at vaginal birth after caesarean: a retrospective cohort study. Int Urogynecol J. 2019;30(10):1747-53.

9. Raisanen S, Vehvilainen-Julkunen K, Cartwright R, Gissler M, Heinonen S. A prior cesarean section and incidence of obstetric anal sphincter injury. Int Urogynecol J. 2013;24(8):1331-9.

10. Hehir M, Fitzpatrick M, Cassidy M, Murphy M, O'Herlihy C. Are women having a vaginal birth after a previous caesarean delivery at increased risk of anal sphincter injury? BJOG: An International Journal of Obstetrics \& Gynaecology. 2014;121(12):1515-20.

11. Richter HE, Brumfield CG, Cliver SP, Burgio KL, Neely CL, Varner RE. Risk factors associated with anal sphincter tear: a comparison of primiparous patients, vaginal births after cesarean deliveries, and patients with previous vaginal delivery. Am J Obstet Gynecol. 2002;187(5):1194-8.

12. Boatin AA, Cullinane F, Torloni MR, Betrán AP. Audit and feedback using the Robson classification to reduce caesarean section rates: a systematic review. Bjog. 2018;125(1):36-42.

13. Sandall J, Tribe RM, Avery L, Mola G, Visser GH, Homer CS, et al. Short-term and long-term effects of caesarean section on the health of women and children. Lancet. 2018;392(10155):1349-57.

14. Ornaghi S, Maraschini A, Donati S. Characteristics and outcomes of pregnant women with placenta accreta spectrum in Italy: A prospective population-based cohort study. PLoS One. 2021;16(6):e0252654.

15. Elvander C, Ahlberg M, Edqvist M, Stephansson O. Severe perineal trauma among women undergoing vaginal birth after cesarean delivery: A population-based cohort study. Birth. 2019;46(2):379-86.

16. Jardine JE, Knight HE, Carroll FE, Gurol-Urganci I. Risk of obstetric anal sphincter injury in women having a vaginal birth after a previous caesarean section: A population-based cohort study. European Journal of Obstetrics \& Gynecology and Reproductive Biology. 2019;236:7-13.

17. Royal College of Obstetricians and Gynaecologists. Birth After Previous Caesarean Birth. Green-top Guideline No 45. 2015.

18. Royal Australian and New Zealand College of Obstetricians and Gynaecologists. Birth after previous caesarean section. 2019.

19. Flood M, Pollock W, McDonald SJ, Davey MA. Accuracy of postpartum haemorrhage data in the 2011 Victorian Perinatal Data Collection: Results of a validation study. Aust N Z J Obstet Gynaecol. 2018;58(2):210-6.

20. Flood MM, McDonald SJ, Pollock WE, Davey MA. Data accuracy in the Victorian Perinatal Data Collection: Results of a validation study of 2011 data. Health Inf Manag. 2017;46(3):113-26.

21. Davey MA, Sloan ML, Palma S, Riley M, King J. Methodological processes in validating and analysing the quality of population-based data: a case study using the Victorian Perinatal Data Collection. Health Inf Manag. 2013;42(3):12-9.

22. Dahlen HG, Ryan M, Homer CS, Cooke M. An Australian prospective cohort study of risk factors for severe perineal trauma during childbirth. Midwifery. 2007;23(2):196-203.

23. Baghestan E, Irgens LM, Børdahl PE, Rasmussen S. Trends in Risk Factors for Obstetric Anal Sphincter Injuries in Norway. Obstetrics \& Gynecology. 2010;116(1):25-34.

24. Jha S, Parker V. Risk factors for recurrent obstetric anal sphincter injury (rOASI): a systematic review and meta-analysis. Int Urogynecol J. 2016;27(6):849-57.

25. Sideris M, McCaughey T, Hanrahan JG, Arroyo-Manzano D, Zamora J, Jha S, et al. Risk of obstetric anal sphincter injuries (OASIS) and anal incontinence: A meta-analysis. Eur J Obstet Gynecol Reprod Biol. $2020 ; 252: 303-12$. 
26. Smith LA, Price N, Simonite V, Burns EE. Incidence of and risk factors for perineal trauma: a prospective observational study. BMC Pregnancy Childbirth. 2013;13:59.

27. Luchristt D, Brown O, Pidaparti M, Kenton K, Lewicky-Gaupp C, Miller ES. Predicting obstetrical anal sphincter injuries in patients who undergo vaginal birth after cesarean delivery. Am J Obstet Gynecol. 2021.

28. Kapoor DS, Thakar R, Sultan AH. Obstetric anal sphincter injuries: review of anatomical factors and modifiable second stage interventions. Int Urogynecol J. 2015;26(12):1725-34.

29. RCOG. The management of third- and fourth-degree perineal tears [Green-top Guideline No. 29]. Royal College of Obstetricians and Gynaecologists; 2015.

30. Verghese TS, Champaneria R, Kapoor DS, Latthe PM. Obstetric anal sphincter injuries after episiotomy: systematic review and meta-analysis. Int Urogynecol J. 2016;27(10):1459-67.

31. Sultan AH, Thakar R, Ismail KM, Kalis V, Laine K, Raisanen SH, et al. The role of mediolateral episiotomy during operative vaginal delivery. Eur J Obstet Gynecol Reprod Biol. 2019;240:192-6.

32. Garmi G, Peretz H, Braverman M, Berkovich I, Molnar R, Salim R. Risk factors for obstetric anal sphincter injury: To prolong or to vacuum? Midwifery. 2016;34:178-82.

33. Deering SH, Carlson N, Stitely M, Allaire AD, Satin AJ. Perineal body length and lacerations at delivery. J Reprod Med. 2004;49(4):306-10.

34. Mankuta DD, Leshno MM, Menasche MM, Brezis MM. Vaginal birth after cesarean section: trial of labor or repeat cesarean section? A decision analysis. Am J Obstet Gynecol. 2003;189(3):714-9.

35. Garretto D, Lin BB, Syn HL, Judge N, Beckerman K, Atallah F, et al. Obesity May Be Protective against Severe Perineal Lacerations. Journal of Obesity. 2016;2016:9376592.

36. Blomberg M. Maternal Body Mass Index and Risk of Obstetric Anal Sphincter Injury. BioMed Research International. 2014;2014:395803.

37. Pergialiotis V, Vlachos D, Protopapas A, Pappa K, Vlachos G. Risk factors for severe perineal lacerations during childbirth. Int J Gynaecol Obstet. 2014;125(1):6-14.

38. Loewenberg-Weisband Y, Grisaru-Granovsky S, Ioscovich A, Samueloff A, Calderon-Margalit R. Epidural analgesia and severe perineal tears: a literature review and large cohort study. J Matern Fetal Neonatal Med. 2014;27(18):1864-9.

39. Gurol-Urganci I, Bidwell P, Sevdalis N, Silverton L, Novis V, Freeman R, et al. Impact of a quality improvement project to reduce the rate of obstetric anal sphincter injury: a multicentre study with a steppedwedge design. BJOG. 2021;128(3):584-92.

40. Safer Care Victoria. Better births for women collaborative. 2021.

41. Regan J, Thompson A, DeFranco E. The influence of mode of delivery on breastfeeding initiation in women with a prior cesarean delivery: a population-based study. Breastfeed Med. 2013;8:181-6.

42. Meddings F, Phipps FM, Haith-Cooper M, Haigh J. Vaginal birth after caesarean section (VBAC): exploring women's perceptions. J Clin Nurs. 2007;16(1):160-7.

43. Dodd JM, Crowther CA. Risks and morbidity associated with previous cesarean section. Womens Health (Lond). 2007;3(1):55-61.

44. Tulandi T, Agdi M, Zarei A, Miner L, Sikirica V. Adhesion development and morbidity after repeat cesarean delivery. Am J Obstet Gynecol. 2009;201(1):56 e1-6. 
CONSORT 2010 Flow Diagram (modified)

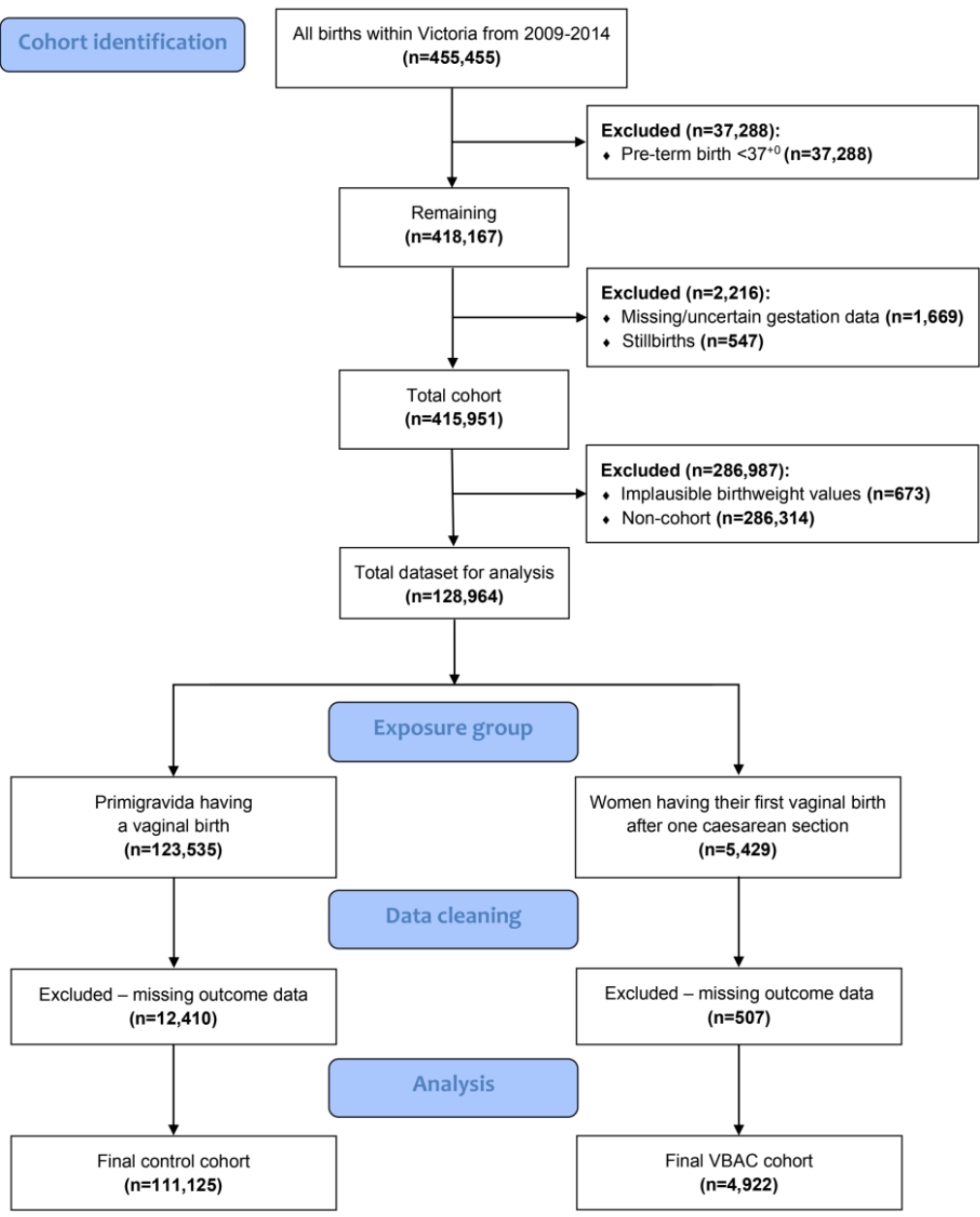

\section{Hosted file}

Tables_VBAC_FINAL.docx available at https://authorea.com/users/436625/articles/538816risk-of-obstetric-anal-sphincter-injury-among-women-who-birth-vaginally-after-a-priorcaesarean-section-a-state-wide-cohort-study 